\title{
Liquid metals: early contributions and some recent developments
}

\author{
C. Regnaut, S. Amokrane \\ Groupe "Physique des Liquides et Milieux Complexes", Faculté des Sciences et Technologie, Université Paris-Est \\ (Créteil), 61 av. du Général de Gaulle, 94010 Créteil Cedex, France
}

Received May 11, 2017, in final form June 29, 2017

\begin{abstract}
We illustrate in this contribution the progress in the theoretical study of liquid metals made in the last decades, starting from the example of liquid gallium and the early work in Jean-Pierre Badiali's group. This was based on the combination of the perturbation theory with pseudo-potentials for the electrons and the liquid state theory for the ions. More recent developments combining ab initio and classical molecular dynamics simulations are finally illustrated on the example of glass forming alloys.
\end{abstract}

Key words: liquid metals, structure, perturbation theory, simulation

PACS: 71.22.+i, 61.20.Gy, 61.20.Ja, 71.15.Pd

\section{Introduction}

Among various fields of research in which Jean Pierre Badiali (JPB) was interested in, we want to emphasize in this contribution some of his early works on liquid metals and discuss some recent developments in this field. In the seventies, Jean Pierre actually devoted a large part of his research to the application of statistical mechanics to the electrochemical interface. He wanted to reach a description of the metal/solution interface at the atomic level in order to develop a multiscale approach of reactivity at the interface. This effort illustrated by an early study of the current-voltage relationship [1] culminated in a series of papers on the contribution of the metal to the properties of the interface, ranging from the capacitance of the ideally polarized electrode [2-5] to the short-range interactions at electrodes coated with conducting polymer films [6]. In the mean time, JPB was convinced that an important task was to accurately describe the bulk solution and the bulk metal by using the best available theories, an effort which should be beneficial to the modelling of the interface itself. In the 1970s, the theory of liquids and metals was rapidly growing. As concerns the liquid state, integral equations, perturbation theories and variational approaches gave a new impulse to the understanding of simple liquids and their mixtures, thanks to the success of the hard sphere (HS) model as a reference system [7]. Regarding the metal studies, the pseudopotential (PP) theory (model or ab initio) was rapidly developing after the first studies in the 1960s [8], allowing one to derive an effective pair potential between the screened ions or pseudoatoms in the metal. For these reasons, JPB decided to use this approach to study the bulk liquid metal. Since we have pursued until nowadays this early work impulsed by JPB, in different contexts, we chose to focus our contribution on these metal studies, including some developments beyond the pair potential approach, that were not present in JPB's work. Actually, liquid and amorphous metals have been the subject of an intense theoretical and experimental activity for decades now, up to forming or being among the major themes of a series of international conferences that started in the mid-sixties ${ }^{1}$ and early-nineties ${ }^{2}$. Understandably, an overview of the related literature would be a very difficult task and we shall not attempt to do this here — see for instance the summaries up to 2002 in [9, 10].

\footnotetext{
${ }^{1}$ Liquid Metals conferences: LM1, Brookhaven, 1966; LAM16, Bonn-Bad Godesberg, 2016

${ }^{2}$ Liquid Matter conferences: LMC1, Lyon, 1990; LMC10, Ljubljana, 2017.
} 
Although mercury or noble metals were more often used in electrochemical cells, JPB focused first on gallium because this metal was extensively studied in the group ${ }^{3}[11]$ and, among all simple metals (i.e., $s-p$ bonded ones), was very puzzling for theorists. Indeed, when compared with other trivalent metallic elements, Ga appears very strange: low melting point temperature $T_{\mathrm{M}}$, exceptional polymorphism and metastability, anomalous liquid structure factor and huge supercooled liquid range of about $-0.5 T_{\mathrm{M}}[12$ 14]. Thus, gallium was, at a first glance, a severe test both for pseudopotential and liquid state theories. After a brief overview of the theoretical methods (section 2), section 3 of this paper is thus devoted to pointing out some of the results early obtained with JBP, to explain qualitatively the structure of liquid $\mathrm{Ga}(1-\mathrm{Ga})$ and justify the relative stability of its numerous solid phases. Then, we briefly survey the state of the art in gallium studies in the 2010s, especially the considerable progress made since the advent of the so-called $a b$ initio molecular dynamics (AIMD) methods, as contrasted with classical molecular dynamics (CMD) with parametrized force fields. As a simple illustration, an example of our own AIMD calculations of the 1-Ga structure will be given. After gallium, JPB focussed in the 1980s on the noble metals. With his co-workers, he used the best available models, i.e., the resonant model [15, 16] or the generalized PP theory (GPT) of Moriarty [17-19] to include the full $d$-band, the $s$ - $d$ hybridization and overlap contributions to derive the effective atomic interactions in noble metals and the corresponding liquid structure and properties [20]. In section 4, we briefly summarize some of these results. Beyond this, the orbital free AIMD method for liquid noble metals [21], will be mentioned. Finally, in connection with the work of JPB and coll. for copper, we show in subsection 4.2 the recent AIMD results on $\mathrm{Cu}$ based alloys by Amokrane et al. [22] in a study that illustrates the efficiency of some modern methods combining AIMD and CMD on the non-trivial case of the glass forming ternary alloy $\mathrm{Cu}_{60} \mathrm{Ti}_{20} \mathrm{Zr}_{20}, \mathrm{a}$ task hardly conceivable with the methods used 40 years ago.

\section{Methodological aspects}

\subsection{The pair potential approach}

Historically, the modern theory of metals emerged from quantum mechanics with the development of interatomic potentials. This was done first in the case of nearly free electron metals for which the electron-ions pseudo potential is sufficiently weak to be treated by perturbation expansions [8, 23, 24]. During nearly thirty years, an intense activity took place to develop interatomic potentials using either first principles PPs or simple local models and to extend the perturbation treatment for $s-p$ metals (see for example [25-27] and [28] for a review) to transition metals, either with full $d$-bands or those with partially field $d$-bands (see [17-[19]). The early work in JPB's group was done in this general framework.

For a given pair potential, the liquid state properties were obtained from the methods of statistical mechanics [7], initially mostly perturbation theory or integral equations for the pair structure, such as the optimized random-phase approximation [29] (ORPA) or the modified hypernetted chain (MHNC) [30, 31], see for example [32,-34]. Later on, the development of the computing power led to a gradual shift from integral equations to simulation, mostly molecular dynamics, see for instance [35-37]. In parallel with the efforts to improve the interatomic potentials, possibly including many-body contributions, the progress made in the calculation of the electronic structure by DFT and a dramatic increase in the computing power led in the early nineties to a radically different approach, as detailed below.

\subsection{Towards the potential energy surface}

The mid-eighties witnessed the development of efficient algorithms for computing the electronic energy for a large number of ionic degrees of freedom, as well as a dramatic increase of the computing power of affordable computers. The effort switched then from the development of semi-analytic firstprinciples interatomic potentials to the purely numerical treatment of the full quantum mechanical manybody electron-ion system. Figure 1 in Moriarty's [38] paper in 2006 provides a clear graphical display of the new situation. The electronic structure is treated in the framework of the Kohn-Sham [39] density functional theory (DFT), in combination either with an extended lagrangian formulation as in

\footnotetext{
${ }^{3}$ Laboratoire de Physique des Liquides et Electrochimie, University Paris 6, France.
} 
the Car Parinello method [40] or by directly computing the forces from the ground state potential energy surface $E\left(\mathbf{R}_{1}, \ldots, \mathbf{R}_{N}\right)$ for each instantaneous configuration of the $N$ ions - see [41] and references therein for the basis of this Born-Oppenheimer molecular dynamics (BOMD) and [42, 44] for actual implementations. The accuracy of these methods benefited from the advent of improved PPs, among which the projector augmented wave (PAW) PP [45, 46] can be as accurate as full-potential approaches [41, 47], such as the LAPW one [48]. They presently form the basis of AIMD simulations in condensed matter physics and materials science. Starting in the early nineties, they have been implemented by different academic groups. Among the various implementations, the self-consistent plane wave (PWscf) fortran code in the open source Quantum-Espresso package [49] has been used in this work and in [22].

In the AIMD of extended systems, pseudo-potentials are still required to avoid costly all-electrons calculations but they are used differently compared to in the interatomic potentials approach, since the DFT treatment of the electronic structure in the total external field created by the ions is not perturbative. According to the review of Kresse in 2002 [50] and references therein, most of the metallic elements can be fairly well described using PAW pseudopotentials. The large number of electrons one needs to consider with accurate PAW PPs, limits, however, the size of the systems that can actually be simulated without large computer resources. The remaining limitations are mostly observed for the low melting point elements. Other limitations also occur due to the approximation made in the DFT such as the local density approximation (LDA) and generalized gradient approximations (GGA).

As a first step, one may also use the orbital free variant of DFT in which only the density is required to evaluate the electronic energy functional. Like for the simple metals, this considerably improved the description of liquid noble metals (see, for instance, the work by Bhuiyan et al. [21] and references therein). The full Kohn-Sham (i.e., with orbitals) calculations are in principle more accurate. However, even with modern computers, the AIMD simulations become impractical if they are to be repeated at different points in the parameters space, as, for example, in the construction of phase diagrams — see e.g., [51] and references therein. One needs then to resort to classical molecular dynamics, with parametrized force fields. (See, for example, [52] for transition metals. Embedded atom models (EAM) potentials for fcc elements are given, for example, in [53] and $n$-body potentials for ternary alloys are reviewed in [54].) In the last section, we thus illustrate the issue of the appropriate combination of $a b$ initio and classical MD on the example of our own work on glass-forming transition metals alloys.

\section{Gallium: from pseudopotentials to ab initio molecular dynamics}

As just mentioned, gallium is known to have numerous structural and thermodynamical peculiarities. The salient ones in the liquid state are: a low melting temperature $T_{\mathrm{M}}=303 \mathrm{~K}$; a density higher than in the stable solid phase; a very large supercooling range of about $150 \mathrm{~K}$; under $257 \mathrm{~K}$ and normal pressure, the supercooled liquid prefers to crystallize in the metastable $\beta$-Ga form [12]; finally, its non-HS-like liquid structure factor with a shoulder in the main peak. Therefore, we first paid attention [11] in 1974 to the difference between a HS-like structure factor $S_{\mathrm{R}}(q)$ as a reference for gallium and the true one $S(q)$. From the PP theory and using the random phase approximation, a very simple expression connects at any temperature this structural difference $\Delta S(q)$ with $\Delta V(q)$, the corresponding difference in $q$-space between the effective interionic interaction and the reference one. For a local PP, the relative change $\Delta S(q) / S(q)$ is linked to the change in the PP by [11]:

$$
\frac{\Delta S(q)}{S(q)}=\frac{S_{\mathrm{R}}(q) \Delta V(q)}{k T} \approx-\frac{1}{k T} S_{\mathrm{R}}(q) f(q)\left[w^{2}(q)-w_{\mathrm{R}}^{2}(q)\right],
$$

where $f(q)$ includes the standard functions of the perturbation theory for nearly free electrons [8]. This function that depends only on the Fermi wavenumber $k_{\mathrm{F}}$ and the valence, induces the so-called Friedel oscillations of $V(r)$ at a large distance. In equation (3.1), the Fourier transform of Ashcroft's empty core model [27] (ECM) is $w(q)=-\frac{4 \pi Z}{\Omega q^{2}} \cos \left(q r_{\mathrm{c}}\right)$, where $\Omega$ is the atomic volume in the liquid, $Z$ is the valence (or effective one) and $r_{\mathrm{c}}$ is the core radius. This approximate expression of $\Delta S(q) / S(q)$ highlights the three pertinent parameters $Z, k_{\mathrm{F}}$ and $r_{\mathrm{c}}$ governing the shape of $S(q)$ and its change from one metal to another. Moreover, since 1-Ga (non-HS-like) and aluminium (HS-like) have the same nominal $Z$ and almost the 
same $k_{\mathrm{F}}$ value, the only difference between these two metals lies in the core radius $r_{\mathrm{c}}$. Unfortunately, we estimated with JPB that $w(q)$ should be known with an accuracy better than 5\%, while the differences found when using various PPs and expressions of the screening function involved in $f(q)$ are much larger. Therefore, it was concluded that if the important structural and thermodynamic features of 1-Ga lie first in its specific PP, both non-locality and accurate liquid state theory methods are required. Using one of the best available non-local model potential developed by Shaw [25, 26] to obtain $V(r)$ and the ORPA to obtain the liquid structure factor, it was shown that Shaw's model can predict the right trends in the structure factors of $\mathrm{Mg}$ and $\mathrm{Al}$ (HS-like) and $\mathrm{Zn}, \mathrm{Ga}, \mathrm{Sn}$ (non-HS-like) [32], but the relative stability of the principal solid phases of Ga could not be fully established without some ad hoc empirical adjustments [55]. On the same basis but with the ECM and ORPA, Hafner and Kahl studied later on the structural trends of the metallic elements in the liquid state over the periodic table in a 3D space [56] (using $Z$, $k_{\mathrm{F}}$ and $r_{\mathrm{c}}$ as variables), following a parent study for their solid structure [57]. They concluded that the complex structure of the light polyvalent liquid metals arise from the interplay of two characteristic distances: the effective diameter $\sigma_{\text {eff }}$ of the repulsive core of $V(r)$ and the wavelength of the Friedel oscillations in the potentials, in full agreement with the studies of JPB and coll.

Unfortunately, other studies and many attempts with different $a b$ initio or models PPs indicate that gallium remains a troublesome metal. Indeed, among the liquid polyvalents metals, some of them although non-HS-like (for instance Ge) are very well described either from the one parameter ECM (Hafner and Kahl [56]) or Shaw's parameter-free model [58]. By contrast, Ga is then poorly described, the ECM being incapable of predicting the stability of the solid form GaI, while Shaw's one does this qualitatively [59, 60].

A few years later, the AIMD methods raised new hopes for accurately describing gallium and nonsimple metals, although the LD and GG approximations seem to have an influence on determining precisely the structure of liquid gallium [50], as well as the relative stability of its crystalline structures at normal and high pressure, at the observed equilibrium volumes [61]. Indeed, previous studies with OPW pseudopotentials [62] can better predict the equilibrium volumes. However, describing all the metastable crystalline $\beta, \gamma$ and $\delta$ phases remains a considerable challenge since a full agreement can be obtained only after very small empirical corrections [55, 59].

The most recent AIMD simulation of liquid gallium we are aware of is that of Chen et al. [63]. The authors simulated a system of $1331 \mathrm{Ga}$ atoms at $T=323 \mathrm{~K}$ at the experimental density $\rho=0.0525 \AA^{-3}$, using a PAW PP and the LDA. They analyzed the bond-orientational order with the aim of identifying the possible source of a shoulder in the first peak of $S(q)$. They concluded that clusters, especially those with fourfold symmetry, could explain this special feature, without excluding the interpretation based on the Friedel oscillations.

Shorter calculations may, however, be useful for a rapid estimation of the pair structure. To illustrate this, we performed a simulation with $N=256$ and a norm conserving (NC) PP with only $Z=3$ electrons (one MD step lasts about $130 \mathrm{~s}$ on our 24 cores machine). The total number of electrons is thus 22.5 times smaller than in a simulation with 1331 ions and $Z=13$ with the available PAW PPs (recall that the computation time increases much faster than the number of electrons). Figure 1 obtained after 1000 steps of equilibration and 1000 steps of accumulation illustrates this possibility to determine a reasonable $g(r)$ with moderate resources (x-ray diffraction (xrd) data are shown for comparison). Partial results after $650 \mathrm{MD}$ steps with a PAW PP showed no significant difference, but this conclusion needs to be confirmed - one MD steps with $N=256$ is about 17 times longer than with the NC PP. The corresponding structure factor shown in figure 2 obtained by Fourier transform of $g(r)$ shows a clear asymmetry of the first peak, though the shoulder is less resolved than in figure 2 of [63] or in the pair potential approach. Among the possible reasons to this, the small system size, the short runs and simple PP used here to speed up the calculation.

Note that in a previous AIMD study on 1-Ga under pressure [64], the analysis of the local geometry and dynamical behavior refutes the hypothesis of $\mathrm{Ga}_{2}$ dimers in the liquid state. It reinforces the interpretation of the shoulder in $S(q)$ for 1-Ga as a result of medium-range order beyond the first shell caused by Friedel oscillations. The authors concluded that their results were consistent with experimental studies [65, 66] in which no evidence was found for any type of "molecular" species at a distance like that in $\alpha$-Ga, nor for any specific type of clustering [66]. This conclusion was drawn from the analysis of the pair structure. A detailed analysis of the local structure, however, requires going beyond the distribution of the distances, as in [63]. 


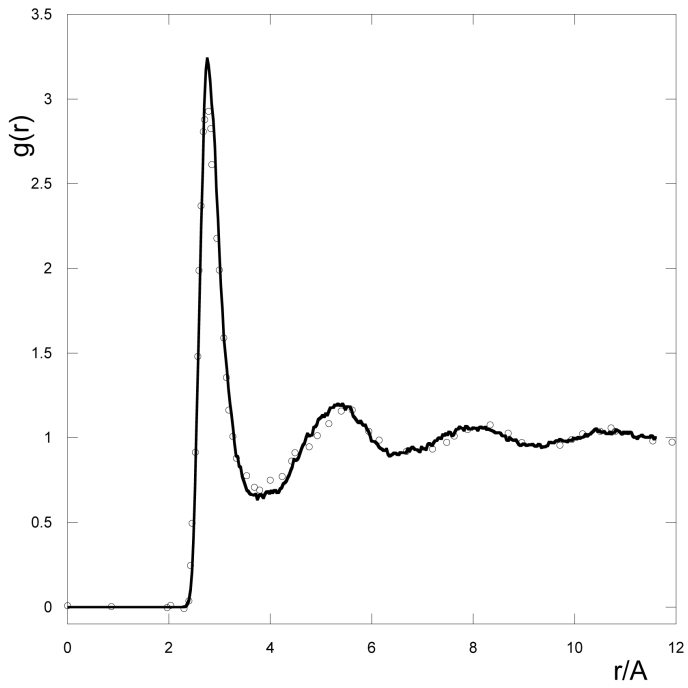

Figure 1. Experimental and theoretical radial distribution function of liquid $\mathrm{Ga}$ at $\mathrm{T}=323 \mathrm{~K}$. Symbols: xrd data [13], full curve: AIMD, this work.

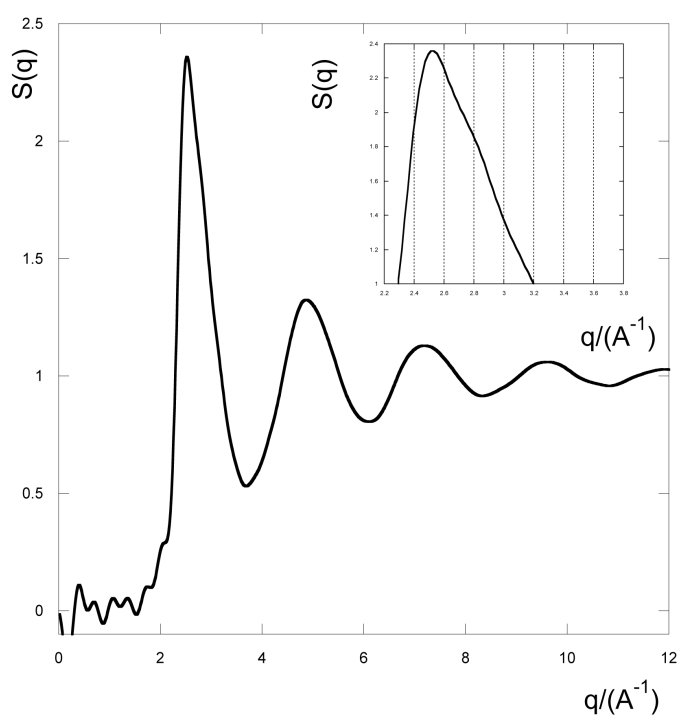

Figure 2. Structure factor of liquid $\mathrm{Ga}$ at $\mathrm{T}=323 \mathrm{~K}$. $S(q)$ is computed from the Fourier transform of the AIMD $g(r)$ in figure 1 Inset shows the first peak on an enlarged scale.

A similar interpretation based on some medium-range order related to the structures beyond the first shell in the radial distribution function (rdf) caused by a cooperation between the short repulsive core and the Friedel oscillations in the pair potential has been discussed in [67]. The situation seems more contrasted in the case of germanium [68].

In summary, AIMD simulations provide a quantitative description of the pair structure of 1-Ga, including a shoulder in the principal peak of $S(q)$, that the pair potential approach is able to reproduce in an effective way. Nonetheless, simulations with large systems are required to definitely assess the precise features in the local structure which are responsible for these collective features, as in [63]. Moreover, AIMD studies in the deep undercooled states are particularly demanding since they require large enough systems to resolve transient structures and a sufficient number of time steps, due to the corresponding slow dynamics.

\section{Beyond simple metals and the interatomic potentials approach}

In this section, we summarize some other results concerning non-simple metals and alloys. Again, we shall not attempt a review of the literature, focusing mostly on the results obtained following JPB's early work. We next present some of our recent extensions of this work.

\subsection{Noble and transition metals}

As underlined in the introduction, JPB's interest in noble metals was motivated by their importance in electrochemistry, in particular, as electrode materials. Transition metals are actually much more difficult to study from first principles pseudopotentials than $s$ - $p$ bonded ones due to the $d$-band contribution. However, the noble metals $\mathrm{Cu}, \mathrm{Ag}, \mathrm{Au}$, have full $d$-band and the theoretical predictions are generally better than for metals having a partially filled $d$-band. In the $1980 \mathrm{~s}$, the best available approaches for noble metals were the resonant model [15] and the generalized pseudopotential theory (GPT) of Moriarty [17.[19]. JPB and colleagues reached the conclusion that the GPT definitely supersedes the resonant model [16] and demonstrated the importance of $s-d$ hybridization and overlap contributions to the effective interaction in noble metals [20] — see also the discussion by Hausleitner et al. [69]. This importance was also underlined in some studies based on the tight binding method [70, 71]. 


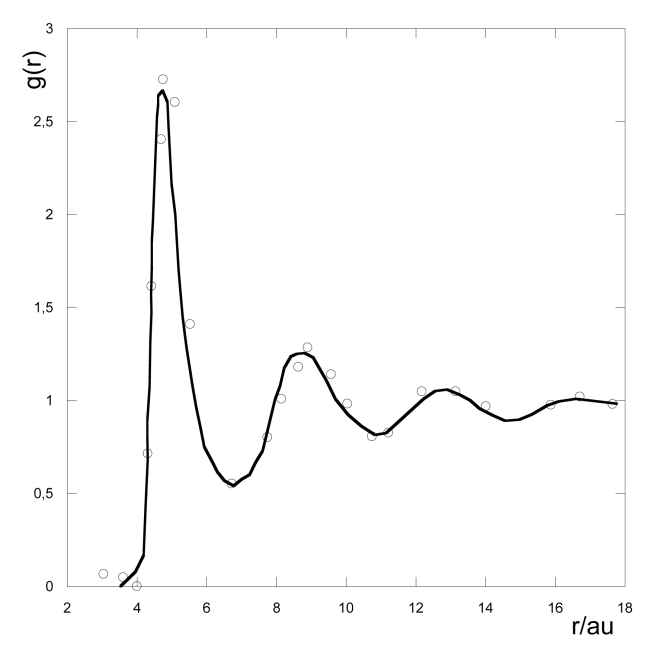

Figure 3. Rdf of pure $\mathrm{Cu}$ near melting. Symbols: xrd data of [72], full curve: parametrized GPT [16]. Distances in atomic units $(0.52917 \AA$ A.)

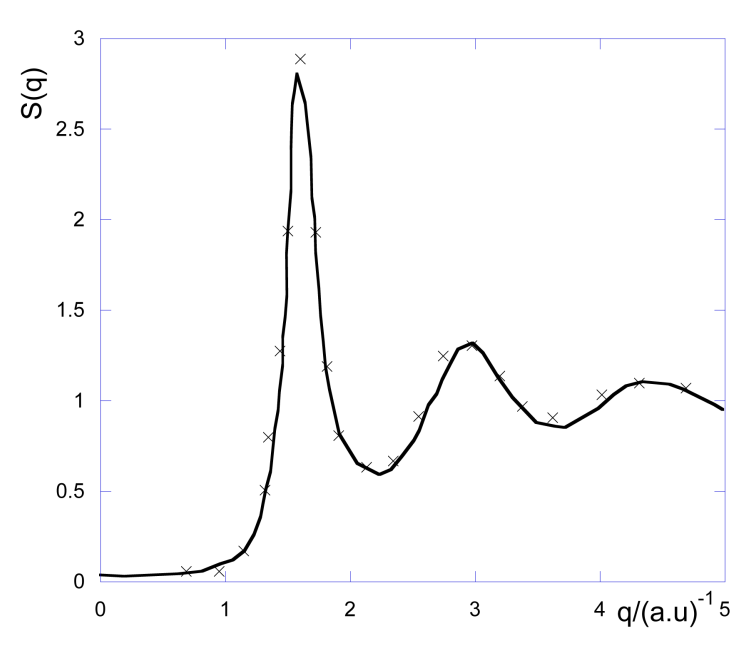

Figure 4. Structure factor of pure $\mathrm{Cu}$ at $\mathrm{T}=1356 \mathrm{~K}$. Symbols: xrd data of [72], full curve: parametrized GPT [16].

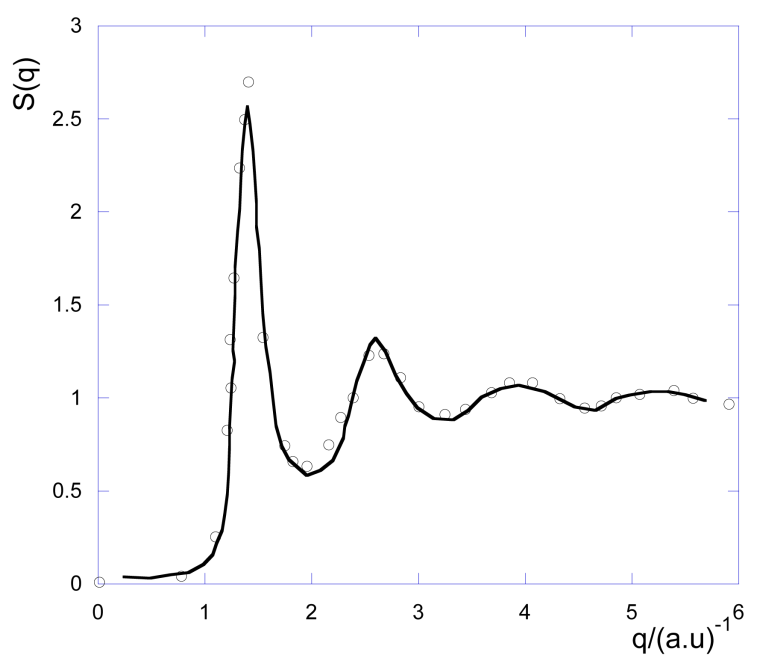

Figure 5. Structure factor of pure Ag at $\mathrm{T}=1234 \mathrm{~K}$. Symbols: $x$ rd data of [72], full curve: parametrized GPT [16].

From a parametrized GPT theory, it was found that the liquid structure of copper is very well described as well as its resistivity and thermodynamical properties. This is illustrated in figures 3 (rdf) and 4 (structure factor). The same approach fairly well describes silver as shown in figure 5, but it fails for $\mathrm{Au}$ [16, 73]. The GPT theory was later extended [74] to transition metals with partially filled $d$-bands. To our knowledge, this approach has not yet been used to study the liquid structure. By contrast, parametrized model potentials such as the Wills-Harisson one allow [75] a simple analysis of some trends in the liquid transition metals [76-78]. Incidentally, using the effective number of $d$ electrons $z_{d}$ of the Wills-Harrisson model improves the calculation of the work function of noble metals [79].

In the last years, the GPT has been considerably improved, by including three and four-body forces. A high degree of accuracy is thus achieved for all transition metals [38, 74, 80]. However, the same period witnessed the rise of the AIMD approach, which, independently of the nature of the metal, bypasses the separation of the total energy $E\left(\mathbf{R}_{1}, \ldots, \mathbf{R}_{N}\right)$ into a sum of $n$-body terms. This point is briefly discussed below (see also the discussion in figure 1 in [38]). 


\subsection{Alloys}

\subsubsection{Liquid alloys}

With mixtures of simple metals, some success has been achieved with interatomic potentials. Numerous studies have been performed to correlate the short and medium range structuring in the liquid and the phase diagram to the effective pair potentials. Much has been done, first in the simple case of the alkali metals (see [28, 33-37] and references therein) and mixtures with polyvalent metals (see, for instance, [81]). These studies either used first principles PPs or a local model to calculate partial liquid structure factors and predict, for instance, the homocoordination preference and demixing [82] — for an overview see [83]. However, the fact that the agreement can be better with simple local models than with non-local norm-conserving PP for pure metals and alloys [84] shows that the situation is not completely clear and raises questions about the use of the corresponding effective pair potentials in classical molecular dynamics [85]. In the same vein, we mention an attempt to go beyond mixtures of simple metals in [86]. On the other hand, the intricacy of the GPT theory for transition metals with partly filled $d$-band makes its implementation difficult, especially for alloys, though several studies have been done in this framework [38, 74, 80].

In the late nineties, numerous AIMD studies of multicomponent alloys of simple metals, less simple and transitions ones were conducted by different groups. A review of this work is beyond the scope of this contribution — see for example [87,-90] and references therein. In the last section, we discuss some problems raised by such simulations.

\subsubsection{Bulk metallic glasses}

In this last section, we illustrate, based on our own work, one last issue in the study of liquid metals, namely the necessity for lengthy calculations to combine classical and $a b$ initio molecular dynamics. The specific $\mathrm{Cu}_{60} \mathrm{Ti}_{20} \mathrm{Zr}_{20}$ system we studied indeed combines several sources of difficulty: ternary alloy of transition metals, studied along a continuous path in the parameters space, with the underlying problem of the transferability of the semi-empirical pair potentials that are unavoidable, say, in a quench at a constant pressure. This system is a prototype of bulk glass forming alloys whose discovery in the late eighties [91] gave a new impetus to the study of amorphous metals. They indeed contrast with the ordinary metallic glasses known from the early sixties [92] that could be elaborated only in the form of very thin ribbons and studied for decades now ${ }^{1,2}$. Since then, such bulk metallic glasses (BMG) have been the subject of numerous studies, due to their unique physical, mechanical and corrosion properties [93- 96 ].

Actually, a variety of combinations of AIMD and CMD simulations have been proposed [97- -101$]$. Among these, the machine learning approach that uses neural networks [102-105] to generate, given a training set of configurations, the weights for the potential energy surface for CMD simulations seems promising. This method may, however, require significant computer resources if the training is made on a large number of AIMD configurations. We illustrate here a particular combination of AIMD and CMD that makes possible the (NPT) simulation of amorphization by melt quenching with moderate computer resources (24 cores in our recent work). As shown in [22] the basic idea is to use AIMD only at the initial and final points of the quench path. It proceeds as follows: (1) perform the $a b$ initio simulations to determine the $\operatorname{rdfs} g_{i j}^{\mathrm{ab}}(r)$ in the liquid (here $T=1600 \mathrm{~K}$ ); (2) determine the parameters denoted by $\left\{a_{i}^{l}\right\}$ of the pair potential by adjustment of the CMD $g_{i j}(r)$ on the $\operatorname{rdfs} g_{i j}^{\mathrm{ab}}(r)$ at $T=1600 \mathrm{~K}$; (3) perform a quench down to $T=300 \mathrm{~K}$ with the CMD simulation; (4) use the last equilibrium configuration so obtained to run the $a b$ initio simulation at $300 \mathrm{~K}$. The AIMD and CMD simulation were made with the Quantum Espresso (QE) [49] and LAMMPS [106] packages, respectively. The CMD simulations were made using the simplified (i.e., without 3-body terms) Stillinger-Weber [107] (SW) potentials used in [108]. The rdfs of $\mathrm{Cu}_{60} \mathrm{Ti}_{20} \mathrm{Zr}_{20}$ were determined from ab initio simulation with ultra-soft pseudopotentials from the QE library. $N=260$ particles $(156+52+52$ atoms for $\mathrm{Cu}, \mathrm{Zr}$, and $\mathrm{Ti}$, respectively) were used, to keep the CPU time in a tolerable range on the 24-core computer used. In these conditions, 1500 steps of accumulation generate, in about 45 days of simulation, reasonably smooth curves for $g_{\mathrm{Cu}-\mathrm{Cu}}, g_{\mathrm{Cu}-\mathrm{Ti}}$, and $g_{\mathrm{Cu}-\mathrm{Zr}}$. With the good scalability with the number of processors of the PWscf code, this restitution time can be reduced to about one week, still with affordable computer resources. Due to the low statistics 


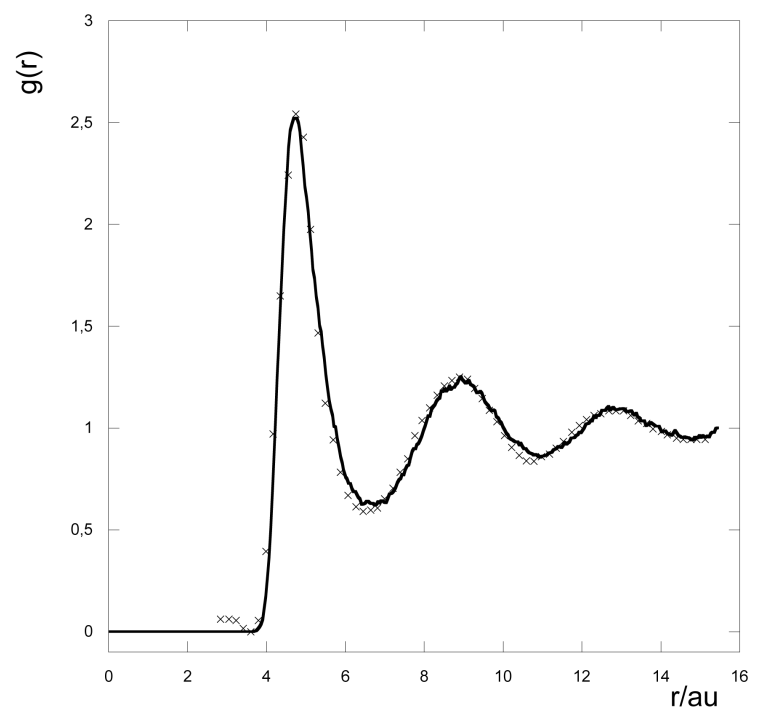

Figure 6. Rdf of pure $\mathrm{Cu}$ at $T=1635 \mathrm{~K}$. Symbols: xrd data of [72] interpolated to $1635 \mathrm{~K}$, full curve: AIMD.

for the minority species, $g_{\mathrm{Ti}-\mathrm{Ti}}, g_{\mathrm{Ti}-\mathrm{Zr}}$, and $g_{\mathrm{Zr}-\mathrm{Zr}}$ in [22] were quite noisy but no significant change was found after additional accumulation - up to 3500 steps — made recently. To illustrate the method for the pure liquids, the rdf of pure liquid copper is shown in figure 6. As already noticed in [22], comparison with figure 3 shows that the GPT theory does almost as well as the AIMD simulation with PAW PP for pure $\mathrm{Cu}$.

In figure 7, we show the partial $\mathrm{rdf}$ for $\mathrm{Cu}$ in the ternary alloy after the quench down to $300 \mathrm{~K}$. $g_{\mathrm{Cu}-\mathrm{Cu}}$ obtained with the SW potentials with the parameters $\left\{a_{i}^{l}\right\}$ (those adjusted at $1600 \mathrm{~K}$ ) are compared to the AIMD ones. The conclusion is that the parameters are here transferable from the liquid to the amorphous alloy, which is an essential feature of the CMD approach with parametrized force fields.

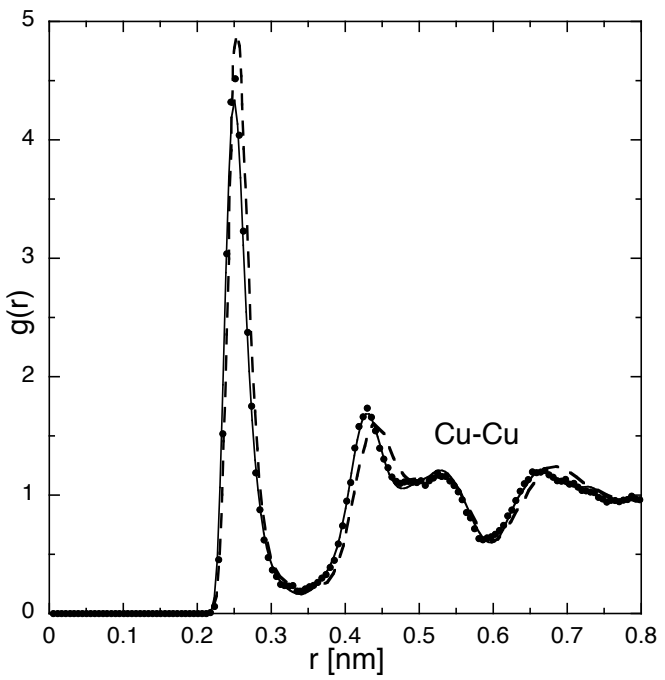

Figure 7. Partial rdf of copper in amorphous $\mathrm{Cu}_{60} \mathrm{Ti}_{20} \mathrm{Zr}_{20}$ at $T=300 \mathrm{~K}$. Full curve: AIMD, dots: CMD with the parameters adjusted at $300 \mathrm{~K}$, dashes: MD simulation [109] with tight-binding potentials for $\mathrm{Cu}_{50} \mathrm{Ti}_{25} \mathrm{Zr}_{25}$.

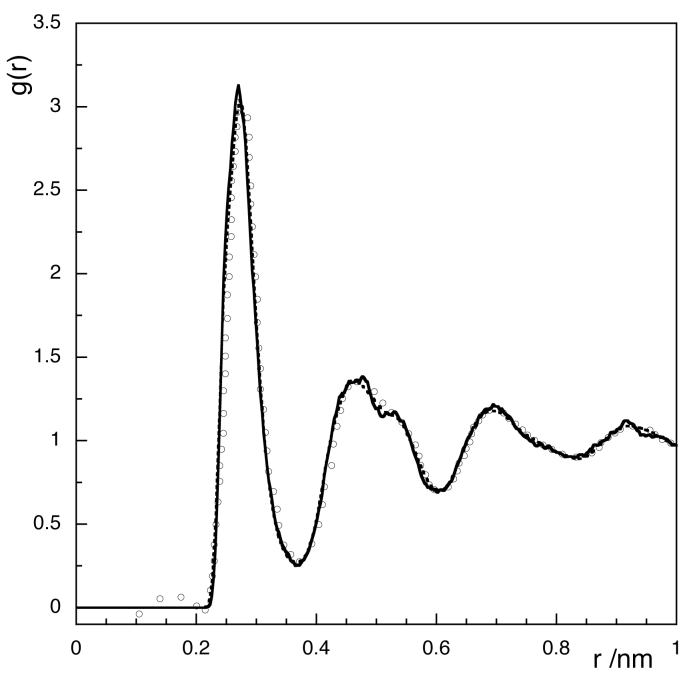

Figure 8. Experimental and simulated total rdf of amorphous $\mathrm{Cu}_{60} \mathrm{Ti}_{20} \mathrm{Zr}_{20}$ at $T=300 \mathrm{~K}$. Symbols: xrd data of Durisin et al. [110], full curve: AIMD, dashes: CMD with the parameters adjusted at $1600 \mathrm{~K}$. 
This is a considerable saving of computer time indeed. The direct comparison with experiment made in figure 8 illustrates the efficiency of the AIMD which justifies the use of the designation of in-silico experiments.

To summarize, the results presented in this section illustrate the possibility of a quantitative determination of the pair structure during the quench by an appropriate combination of $a b$ initio and classical molecular dynamics simulations. They again underline the importance of developing thoroughly tested parametrized force fields, in order to keep simulations convenient enough for a quantitative study of the behavior of complex materials.

\section{Conclusion}

In conclusion, this overview of the work done during four decades, especially the studies that followed from the initial impulse given by JPB, shows a considerable progress made in the search of quantitative methods for studying metallic elements. A gradual switch from approximate semi-analytic methods to the accurate numerical treatment of the quantum many-body electron-ion problem has been emphasized. Nowadays, the most efficient simulation methods seem to be the ones that use classical MD simulations whose force fields are parametrized using the data obtained by AIMD simulations. The resulting approach is flexible and accurate enough to enable a reliable exploration of the parameters space, as required, for example, in the construction of phase diagrams. Our recent work in this domain pursued the hope of JeanPierre Badiali to describe complex and interfacial systems starting from a true microscopic approach, with well controlled theoretical methods.

\section{References}

1. Badiali J.P., Gabrielli C., J. Chim. Phys., 1972, 69, 1725, doi $10.1051 /$ jcp/1972691725

2. Badiali J.P., Goodisman J., J. Electroanal. Chem. Interfacial Electrochem., 1975, 65, 523, doi $10.1016 / 0368-1874(75) 85141-0$

3. Rosinberg M.L., Badiali J.P., Goodisman J., J. Electrochem. Soc., 1982, 129, C138.

4. Amokrane S., Badiali J.P., J. Electroanal. Chem. Interfacial Electrochem., 1989, 266, 21, doi $10.1016 / 0022-0728(89) 80212-8$

5. Amokrane S., Badiali J.P., In: Modern Aspects of Electrochemistry, Bockris J., Conway B.E., White R.E. (Eds.), Plenum, New York, 1992, 1-95.

6. Vorotyntsev M.A., Badiali J.P., Electrochim. Acta, 1994, 39, 289, doi 10.1016/0013-4686(94)80064-2

7. Hansen J.P., MacDonald I.R., Theory of Simple Liquids, Academic Press, London, 1986.

8. Harrison W.A., Pseudopotentials in the Theory of Metals, Benjamin, New York, 1966.

9. Cowlam N., J. Non-Cryst. Solids, 1996, 205-207, 567, doi 10.1016/S0022-3093(96)00276-1.

10. Hensel F., J. Non-Cryst. Solids, 2002, 312-314, 1, doi 10.1016/S0022-3093(02)01640-X.

11. Badiali J.P., Regnaut C., Phys. Status Solidi B, 1974, 63, 555, doi:10.1002/pssb.2220630216

12. Defrain A., J. Chim. Phys., 1977, 74, 851, doi $10.1051 /$ jcp/1977740851

13. Bizid A., Cortes R., Defrain A., Regnaut C., Bellissent R., Tourand G., J. Chim. Phys., 1980, 77, 779, doi $10.1051 / \mathrm{jcp} / 1980770779$.

14. Badiali J.P., Bosio L., Cortès R., Bondot P., Loupias G., Petiau J., J. Phys. Colloques, 1980, 41, C8-211, doi $10.1051 /$ jphyscol:1980854

15. Dagens J., J. Phys. F: Met. Phys., 1976, 6, 1801, doi 10.1088/0305-4608/6/10/012

16. Regnaut C., Fusco E., Badiali J.P., Phys. Status Solidi B, 1983, 120, 373, doi: $10.1002 / p s s b .2221200138$

17. Moriarty J.A., Phys. Rev. B, 1970, 1, 1363, doi:10.1103/PhysRevB.1.1363

18. Moriarty J.A., Phys. Rev. B, 1977, 16, 2537, doi:10.1103/PhysRevB.16.2537

19. Moriarty J.A., Phys. Rev. B, 1982, 26, 1754, doi:10.1103/PhysRevB.26.1754

20. Regnaut C., Fusco E., Badiali J.P., Phys. Rev. B, 1985, 31, 771, doi:10.1103/PhysRevB.31.771

21. Bhuiyan G.M., Gonzáles L.E., Gonzáles D.J., Condens. Matter Phys., 2012, 15, 33604, doi $10.5488 /$ CMP.15.33604

22. Amokrane S., Ayadim A., Levrel L., J. Appl. Phys., 2015, 118, 194903, doi $10.1063 / 1.4935876$

23. Cohen M.H., Heine V., Phys. Rev., 1961, 122, 1821, doi:10.1103/PhysRev.122.1821

24. Ashcroft N.W., Langreth D.C., Phys. Rev., 1967, 155, 682, doi $10.1103 /$ PhysRev.155.682

25. Shaw R.W. (Jr.), Phys. Rev., 1968, 174, 769, doi 10.1103/PhysRev.174.769. 
26. Shaw R.W. (Jr.), J. Phys. C: Solid State Phys., 1969, 2, 2335, doi $10.1088 / 0022-3719 / 2 / 12 / 318$.

27. Ashcroft N.W., Phys. Lett., 1966, 23, 48, doi 10.1016/0031-9163(66)90251-4

28. Hafner J., From Hamiltonians to Phase Diagrams. The Electronic and Statistical-Mechanical Theory of $s p$ Bonded Metals and Alloys, Springer-Verlag, Berlin, Heidelberg, 1987.

29. Andersen H.C., Chandler D., Weeks J.D., J. Chem. Phys., 1972, 56, 3812, doi 10.1063/1.1677784.

30. Lado F., Phys. Rev. A, 1973, 8, 2548, doi 10.1103/PhysRevA.8.2548

31. Rosenfeld Y., Ashcroft N.W., Phys. Rev. A, 1979, 20, 1208, doi:10.1103/PhysRevA.20.1208

32. Regnaut C., Badiali J.P., Dupont M., Phys. Lett. A, 1979, 74, 245, doi:10.1016/0375-9601(79)90783-7

33. Kahl G., Hafner J., Phys. Chem. Liq., 1988, 17, 267, doi:10.1080/00319108808078563

34. Kahl G., Phys. Rev. A, 1991, 43, 822, doi:10.1103/PhysRevA.43.822.

35. Hoshino K., van Wering J.J., J. Phys. F: Met. Phys., 1988, 18, L23, doi:10.1088/0305-4608/18/3/001

36. Canales M., González D.J., González L.E., Padró J.A., Phys. Rev. E, 1998, 58, 4747, doi $10.1103 /$ PhysRevE.58.4747.

37. Wax J.F., Jakse N., Charpentier I., Physica B, 2003, 337, 154, doi:10.1016/S0921-4526(03)00398-3.

38. Moriarty J.A., Benedict L.X., Glosli J.N., Hood R.Q., Orlikowski D.A., Patel M.V., Söderlind P., Streitz F.H., Tang M., Yang L.H., J. Mater. Res., 2006, 21, 563, doi:10.1557/jmr.2006.0070

39. Kohn W., Sham L.J., Phys. Rev., 1965, 140, A1133, doi 10.1103/PhysRev.140.A1133.

40. Car R., Parinello M., Phys. Rev. Lett., 1985, 55, 2471, doi:10.1103/PhysRevLett.55.2471.

41. Martin R.M., Electronic Structure, Cambridge University Press, Cambridge, 2005.

42. Kresse G., Hafner J., Phys. Rev. B, 1993, 47, 558(R), doi 10.1103/PhysRevB.47.558

43. Kresse G., Hafner J., Phys. Rev. B, 1993, 48, 13115, doi:10.1103/PhysRevB.48.13115

44. Kresse G., Furthmüller J., Phys. Rev. B, 1996, 54, 11169, doi:10.1103/PhysRevB.54.11169.

45. Blöchl P.E., Phys. Rev. B, 1994, 50, 17953, doi 10.1103/PhysRevB.50.17953

46. Kresse G., Joubert D., Phys. Rev. B, 1999, 59, 1758, doi 10.1103/PhysRevB.59.1758

47. Holzwarth N.A.W., Matthews G.E., Dunning R.B., Tackett A.R., Zeng Y., Phys. Rev. B, 1997, 55, 2005, doi $10.1103 /$ PhysRevB.55.2005.

48. Singh D.J., Nordström L., Planewaves, Pseudopotentials and the LAPW Method, Springer, New York, 2005.

49. Giannozzi P., Baroni S., Bonini N., Calandra M., Car R., Cavazzoni C., Ceresoli D., Chiarotti G.L., Cococcioni M., Dabo I., et al., J. Phys.: Condens. Matter, 2009, 21, 395502, doi 10.1088/0953-8984/21/39/395502

50. Kresse G., J. Non-Cryst. Solids, 2002, 312-314, 52, doi $10.1016 /$ S0022-3093(02)01649-6

51. Harvey J.P., Gheribi A.E., Chartrand P., Phys. Rev. B, 2012, 86, 224202, doi $10.1103 /$ PhysRevB.86.224202

52. Alemany M.M.G., Diéguez O., Rey C., Gallego L.J., Phys. Rev. B, 1999, 60, 9208, doi $10.1103 /$ PhysRevB.60.9208.

53. Sheng H.W., Kramer M.J., Cadien A., Fujita T., Chen M.W., Phys. Rev. B, 2011, 83, 134118 , doi $10.1103 /$ PhysRevB.83.134118.

54. Li J.H., Dai Y., Dai X.D., Intermetallics, 2012, 31, 292, doi $10.1016 /$ j.intermet.2012.05.018

55. Regnaut C., Badiali J.P., Dupont M., J. Phys. Colloques, 1980, 41, C8-603, doi $10.1051 /$ jphyscol:19808153

56. Hafner J., Kahl G., J. Phys. F: Met. Phys., 1984, 14, 2259, doi $10.1088 / 0305-4608 / 14 / 10 / 006$

57. Hafner J., Heine V., J. Phys. F: Met. Phys., 1983, 13, 2479, doi: 10.1088/0305-4608/13/12/008

58. Regnaut C., Bretonnet J.L., Phys. Rev. B, 1988, 38, 10922, doi 10.1103/PhysRevB.38.10922

59. Dupont M., Thèse, Université Paris 6, 1976.

60. Regnaut C., Thèse, Université Paris 6, 1981 .

61. Bernasconi M., Chiarotti G.L., Tosatti E., Phys. Rev. B, 1995, 52, 9988, doi 10.1103/PhysRevB.52.9988

62. Hafner J., Hittmair O., Österr. Akad. Wiss. Math. Naturwiss., 1975, 4, 1.

63. Chen L.Y., Tang P.H., Wu T.M., J. Chem. Phys., 2016, 145, 024506, doi:10.1063/1.4955305

64. Yang J., Tse J.S., Iitaka T., J. Chem. Phys., 2011, 135, 044507, doi 10.1063/1.3615936

65. Ascarelli P., Phys. Rev., 1966, 143, 36, doi:10.1103/PhysRev.143.36

66. Nield V.M., McGreevy R.L., Tucker M.G., J. Phys.: Condens. Matter, 1998, 10, 3293, doi $10.1088 / 0953-8984 / 10 / 15 / 006$

67. Wu T.M., AIP Conf. Proc., 2013, 1518, 411, doi $10.1063 / 1.4794605$

68. Takeuchi N., Garzón I.L., Phys. Rev. B, 1994, 50, 8342, doi:10.1103/PhysRevB.50.8342.

69. Hausleitner C., Kahl G., Hafner J., J. Phys.: Condens. Matter, 1991, 3, 1589, doi $10.1088 / 0953-8984 / 3 / 11 / 017$

70. Manh D.N., Mayou D., Pasturel A., Cyrot-Lackmann F., J. Phys. F: Met. Phys., 1985, 15, 1911, doi $10.1088 / 0305-4608 / 15 / 9 / 010$

71. Phuong L.D., Pasturel A., Manh D.N., J. Phys: Condens. Matter, 1993, 5, 1901, doi $10.1088 / 0953-8984 / 5 / 13 / 008$

72. Waseda Y., The Structure of Non-Crystalline Materials, McGraw-Hill, New York, 1980.

73. Fusco E., Thèse, Université Paris 6, 1983. 
74. Moriarty J.A., Phys. Rev. B, 1990, 42, 1609, doi:10.1103/PhysRevB.42.1609

75. Wills J.M., Harrison W.A., Phys. Rev. B, 1983, 28, 4363, doi:10.1103/PhysRevB.28.4363

76. Regnaut C., Z. Phys. B: Condens. Matter, 1989, 76, 179, doi $10.1007 / B F 01312682$.

77. Hausleitner C., Hafner J., J. Phys. F: Met. Phys., 1988, 18, 1013, doi: 10.1088/0305-4608/18/6/007

78. Hausleitner C., Hafner J., J. Phys. F: Met. Phys., 1988, 18, 1025, doi $10.1088 / 0305-4608 / 18 / 6 / 008$

79. Russier V., Regnaut C., Badiali J.P., Z. Phys. Chem., 1988, 156, 489, doi $10.1524 /$ zpch.1988.156.Part_2.489

80. Moriarty J.A., Widom M., Phys. Rev. B, 1997, 56, 7905, doi 10.1103/PhysRevB.56.7905

81. Gonzáles L.E., Gonzáles D.J., Silbert M., J. Non-Cryst. Solids, 1996, 205-207, 443, doi $10.1016 / \mathrm{S} 0022-3093(96) 00257-8$

82. Takhtoukh A., Grosdidier B., Hellal S., Regnaut C., J. Phys. Conf. Ser., 2008, 98, 042003 , doi $10.1088 / 1742-6596 / 98 / 4 / 042003$

83. Singh R.N., Sommer F., Rep. Prog. Phys., 1997, 60, 57, doi 10.1088/0034-4885/60/1/003

84. Fiolhais C., Perdew J.P., Armster S.Q., MacLaren J.M., Brajczewska M., Phys. Rev. B, 1995, 51, 14001, doi $10.1103 /$ PhysRevB.51.14001

85. Hellal S., Nigon M., Takhtoukh A., Grosdidier B., Regnaut C., J. Non-Cryst. Solids, 2007, 353, 3467, doi $10.1016 /$ j.jnoncrysol.2007.05.123.

86. Khaleque M.A., Bhuiyan G.M., Sharmin S., Rashid R.I.M.A., Mujibur Rahman S.M., Eur. Phys. J. B, 2002, 26, 319 , doi $10.1140 /$ epjb/e20020095

87. Costa Cabral B.J., Martins J.L., J. Chem. Phys., 1999, 111, 5067, doi $10.1063 / 1.479762$

88. Senda Y., Shimojo F., Hoshino K., J. Phys. Soc. Jpn., 1998, 67, 2753, doi 10.1143/JPSJ.67.2753

89. Gonzáles D.J., Gonzáles L.E., López J.M., Stott M.J., Europhys. Lett., 2003, 62, 42, doi:10.1209/epl/i2003-00357-4

90. Jakse N., Pasturel A., Phys. Rev. B, 2008, 78, 214204, doi 10.1103/PhysRevB.78.214204

91. Inoue A., Ohtera K., Kita K., Masumoto T., Jpn. J. Appl. Phys., 1988, 27, L2248, doi:10.1143/JJAP.27.L2248

92. Klement W., Willens R.H., Duwez P., Nature, 1960, 187, 869, doi $10.1038 / 187869$ b0

93. Niinomi M., Sci. Technol. Adv. Mater., 2003, 4, 445, doi 10.1016/j.stam.2003.09.002.

94. Zhang B., Zhao D.Q., Pan M.X., Wang W.H., Greer A.L., Phys. Rev. Lett., 2005, 94, 205502, doi 10.1103/PhysRevLett.94.205502

95. Trexler M.M., Thadhani N.N., Prog. Mater. Sci., 2010, 55, 759, doi 10.1016/j.pmatsci.2010.04.002

96. Gong P., Yao K., Wang X., Shao Y., Adv. Eng. Mater., 2013, 15, 691, doi 10.1002/adem.201200391.

97. Ercolessi F., Adams J.B., Europhys. Lett., 1994, 26, 583, doi 10.1209/0295-5075/26/8/005.

98. Tafipolsky M., Schmid R., J. Phys. Chem. B, 2009, 113, 1341, doi 10.1021/jp807487f

99. Zope R.R., Mishin Y., Phys. Rev. B, 2003, 68, 024102, doi $10.1103 /$ PhysRevB.68.024102

100. Li J.H., Dai X.D., Liang S.H., Tai K.P., Kong Y., Liu B.X., Phys. Rep., 2008, 455, 1, doi $10.1016 /$ j.physrep.2007.09.004

101. Jakse N., Pasturel A., Comput. Mater. Sci., 2010, 49, S272, doi:10.1016/j.commatsci.2010.03.022

102. Behler J., Parrinello M., Phys. Rev. Lett., 2007, 98, 146401, doi:10.1103/PhysRevLett.98.146401.

103. Eshet H., Khaliullin R.Z., Kühne T.D., Behler J., Parrinello M., Phys. Rev. B, 2010, 81, 184107, doi $10.1103 /$ PhysRevB.81.184107.

104. Behler J., J. Chem. Phys., 2016, 145, 170901, doi $10.1063 / 1.4966192$

105. Artrith N., Urban A., Comput. Mater. Sci., 2016, 114, 135, doi $10.1016 /$ j.commatsci.2015.11.047

106. Plimpton S., J. Comput. Phys., 1995, 117, 1, doi:10.1006/jcph.1995.1039

107. Stillinger F.H., Weber T.A., Phys. Rev. B, 1985, 31, 5262, doi $10.1103 /$ PhysRevB.31.5262

108. Han X.J., Teichler H., Phys. Rev. E, 2007, 75, 061501, doi 10.1103/PhysRevE.75.061501

109. Senturk Dalgic S., Celtek M., EPJ Web Conf., 2011, 15, 03008, doi $10.1051 /$ epjconf/20111503008

110. Durišin J., Balga D., Saksl K., Pietriková A., J. Alloys Compd., 2014, 608, 241, doi $10.1016 /$ j.jallcom.2014.04.103 


\section{Рідкі метали: ранні роботи та деякі недавні дослідження}

\section{К. Реньо, С. Амокран}

Група “Фізика рідин і складних середовищ”, факультет природничих і технічних наук, Університет Парі-Ест, Франція

В цій статті ми обговорюємо розвиток теоретичних досліджень рідких металів, виконаних за останні десятиліття, починаючи з прикладу рідкого галію і ранніх робіт в групі Жана-П'єра Бадіалі. Вони грунтуються на поєднанні теорії збурень з псевдопотенціалами для електронів і теорії рідкого стану для іонів. Наприкінці, на прикладі склоутворюючих розплавів, проілюстровано пізніші дослідження, які поєднують метод $a b$ initio та класичний метод молекулярної динаміки.

Ключові слова: рідкі метали, структура, теорія збурень, моделювання 